\title{
Nutrients and brain health: an overview
}

\author{
Jeremy P. E. Spencer
}

Received: 21 July 2009/ Accepted: 3 August 2009/Published online: 3 September 2009

(C) Springer-Verlag 2009

Over the last 10-20 years, a huge research effort has been afforded to the impact that the diet plays in preventing both cardiovascular disease and the development of various cancers. However, over the same period there has been relatively little research conducted into the role diet plays in both normal and abnormal brain ageing. Due to significant advances in medical science over the last century, there has been a gradual but highly significant increase in human life span, which is particularly apparent in developed nations. On the surface, an extension of the human life span appears to be a great achievement. However, with this increase comes a potential problem. As people age beyond 70 years they become increasingly susceptible to chronic and extremely debilitating brain diseases, most notably Alzheimer's and Parkinson's disease. Ageing is associated with many chronic neurodegenerative diseases. However, the precise cause of the neuronal degeneration underlying these diseases, and indeed normal brain ageing remain unclear. It is thought that several cellular and molecular events are involved, including increases in oxidative stress, impaired mitochondria function, activation of neuronal apoptosis, the deposition of aggregated proteins and excitotoxicity. Thus far, the majority of existing drug treatments for neurodegenerative disorders are unable to prevent the underlying degeneration of neurons and consequently there is a desire to develop alternative therapies capable of preventing the progressive loss of specific neuronal populations. Since the neuropathology of many neurodegenerative diseases has been linked to increases in brain oxidative stress, historically, strong efforts have been

J. P. E. Spencer $(\bowtie)$

Molecular Nutrition Group, School of Chemistry, Food and Pharmacy, University of Reading, Reading RG6 6AP, UK e-mail: j.p.e.spencer@reading.ac.uk directed at exploring antioxidant strategies to combat neuronal damage. Recently, there has been intense interest in the neuroprotective effects of a group of plant secondary metabolites known as flavonoids, which are powerful antioxidants in vitro. Furthermore, there has also been interest in potential beneficial effects of specific polyunsaturated lipids, mainly due to the fact that the brains cellular architecture is particularly rich in these lipids.

The notion that diet influences the functioning of the brain may at first appear obvious. For example, the brain has a very high-energy demand and, as such, utilises a large proportion of the dietary intake of carbohydrates in order to function effectively. Furthermore, macronutrients such as lipids are vital components of both neurons and glial cells and their profile (saturated or un-saturated) has been proposed to play a huge role in brain function. However, dietary lipids, such as polyunsaturated fatty acids, are also thought to play a much wider role in supporting optimum brain function. For example, they are capable of maintaining the optimal function of cholinergic neurons arising from the basal forebrain and terminating in the cortex and hippocampus [7]. Consequently, they may hold the potential to prevent the cognitive decline that occurs during normal ageing and in Alzheimer's disease. However, it is less obvious how other dietary-derived nutrients or nonnutrient components may impact on the functioning of the brain. Despite this, a large number of dietary intervention studies in humans [1] and animals [3], in particular those using foods and beverages derived from Vitis vinifera (grape), Camellia sinensis (tea), Theobroma cacao (cocoa) and Vaccinium spp (blueberry) have demonstrated beneficial effects on human vascular function and on improving memory and learning [1-3]. While such foods and beverages differ greatly in chemical composition, macro- and micronutrient content and caloric load per serving, they 
have in common that they are amongst the major dietary sources of a group of phytochemicals called flavonoids.

It appears that these low molecular weight, non-nutrient components are able to impact upon memory through their ability to exert effects directly on the brains innate architecture for memory. Flavonoids are now thought to be capable of improving cognitive performance via an ability to interact with neuronal and glial signalling pathways crucial in inducing synaptic plasticity [4]. For example, flavonoids may activate both the ERK and Akt pathways leading ultimately to an increase in the number of, and strength of, connections between neuronal populations. However, their interaction with these pathways has wider relevance, as such signalling cascades are also responsible for determining the fate of neurons following interactions with neurotoxins and inflammatory mediators [4]. As such, nutrients which interact with these pathways may also be capable of reducing the neurodegenerative injury associated with major brain diseases and stroke. Indeed, the interactions of the green tea polyphenol, (-)-epigallocatechin-3-gallate (EGCG) with diverse signalling pathways is known to mediate its neuroprotective activity, particularly that associated with Parkinson's disease [6].

The well characterised effects of both dietary phytochemicals and lipids on endothelial function and peripheral blood flow may also hint at an exciting future application of these nutrients on brain health. Nutrient induced vascular effects are potentially significant as increased cerebrovascular function and increased brain blood flow are known to facilitate adult neurogenesis in the hippocampus [5]. Endothelial cells lining the blood vessels of the brain are critical components of the neural stem cell niche, releasing soluble factors that regulate neural stem cell proliferation and differentiation. Furthermore, ageing is known to impair vascularisation, endothelial function and decreases endothelial progenitor cell recruitment, which could adversely affect neurogenesis. Therefore, the influence of dietary agents on angiogenesis and the production of vascular derived factors are also likely to influence neurogenesis. If such effects prove possible, then diet would have the potential to not only slow the progression of neurodegeneration and cognitive decline, but also to potentially reverse disease and cognitive impairment via the re-population of neurons in the hippocampus.

Finally, the potential impact of diet on health care costs should not be ignored. Dementia costs to the UK alone have been estimated to be $£ 17$ billion per annum. If scientists could develop a treatment that would reduce severe cognitive impairment in older people by just $1 \%$ per year, this would cancel out all estimated increases in the longterm care costs due to our ageing population (Alzheimer's Research Trust). Beyond this, there is also intense interest in the development of drugs capable of enhancing memory and learning, both in adults and children, and there is a strong possibility that in the future specific nutrients, in particular flavonoids, might act as precursors for the development of a new generation of memory enhancing drugs. As such, the current series of reviews in this issue are extremely timely and both highlight the current thinking in the field and outline future directions for research in the area.

\section{References}

1. Macready AL, Kennedy OB, Ellia JA, Williams CM, Spencer JPE, Butler LT (2009) Flavonoids and cognitive function: a review of human randomized controlled trial studies and recommendations for future studies. Genes Nutr 4. doi:10.1007/s12263-009-0135-4

2. Maher P (2009) Modulation of multiple pathways involved in the maintenance of neuronal function during aging by fisetin. Genes Nutr 4. doi:10.1007/s12263-009-0142-5

3. Rendeiro C, Spencer JPE, Vauzour D, Butler LT, Williams CM (2009) The impact of flavonoids on spatial memory in rodents: from behaviour to underlying hippocampal mechanisms. Genes Nutr 4. doi:10.1007/s12263-009-0137-2

4. Spencer JPE (2009) Flavonoids and brain health: multiple effects underpinned by common mechanisms. Genes Nutr 4. doi: 10.1007/s12263-009-0136-3

5. Stangl D, Thuret S (2009) Impact of diet on adult hippocampal neurogenesis. Genes Nutr 4. doi:10.1007/s12263-009-0134-5

6. Weinreb O, Amit T, Mandel S, Youdim MBH (2009) Neuroprotective molecular mechanisms of (-)-epigallocatechin-3-gallate: a reflective outcome of its antioxidant, iron chelating and neuritogenic properties. Genes Nutr 4. doi:10.1007/s12263-009-0143-4

7. Willis LM, Shukitt-Hale B, Joseph JA (2009) Dietary polyunsaturated fatty acids improve cholinergic transmission in the aged brain. Genes Nutr 4. doi:10.1007/s12263-009-0141-6 